MKG-Chirurg 2008 · 1:174-175

DOI 10.1007/s12285-008-0059-9

Online publiziert: 25. Oktober 2008

(c) Springer Medizin Verlag 2008

\author{
B.F. Connell \\ Santa Ana, California, USA
}

\title{
Zeitgemäße Konzepte in der ästhetischen Gesichtschirurgie
}

nen, welche Nasenform das Gesicht ansprechend aussehen lässt, welche Proportionen am angenehmsten $\mathrm{zu}$ betrachten sind und welches die entscheidenden Merkmale der Augen, des Gesichts, der Stirn und des Halses für ein in sich stimmiges und schönes Gesamterscheinungsbild sind.

\section{() Der wichtigste Teil des Verfahrens zur Minimierung von Narben ist die Technik der Hautexzision}

Die verjüngende Wiederherstellung erfordert Detailplanung, unterstützt durch Einsichtnahme in Fotografien früherer Jahre, als der Patient mit seiner Erscheinung am glücklichsten war (• Abb. 1). Ästhetisch unbefriedigende Narben sind glücklicherweise sehr selten, wenn der Eingriff durch geschickte Chirurgen vorgenommen wurde, die in der Gesichtsanatomie erfahren sind und sich die Zeit nehmen, einerseits die Zeichen eines chirurgischen Eingriffs $\mathrm{zu}$ verhindern und andererseits ansprechende und jugendliche Konturen wiederherzustellen. Bei jüngeren Patienten mit Entstellungen des Gesichts, der Nase und des Halses kann die Chirurgie Schönheit unabhängig von der ästhetischen Ausgangssituation herausbilden. $\mathrm{Zu}$ den nicht akzeptablen Ergebnissen eines Faceliftings zählen sichtbare Narben, eine entstellende Erscheinung, Tragusverformungen, wie keine Endungen oder eine unangemessene Größe, neben fehlgesetzten Ohrläppchen und versetzten Haaren. Auffällige Narben sind in der Regel zurückzuführen auf den nicht adäquaten Einschnittort und Farbabgleich, gekrümmte oder gerade Einschnitte, Fehler bei der Nahttechnik, Unwissen über die normale Erscheinung eines jugendhaften Gesichts, Ohren und anderer Merkmale, Hautspannung, Trauma und Nekrose, postoperative Schwellungen sowie Fehler bei der Nachversorgung.
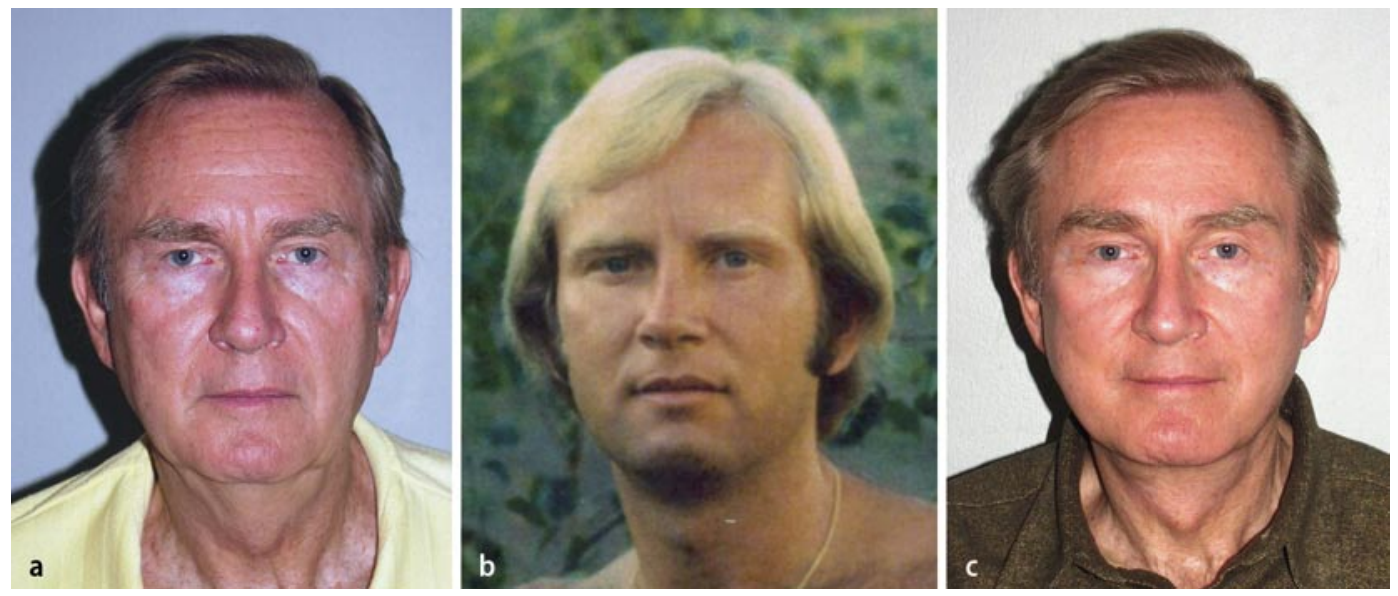

Abb. $1 \varangle$ Fotografien des Patienten im Alter von, a 64 Jahren (25.04.2007), b 31 Jahren, c 64 Jahren 9 Monate postoperativ (08.02.2008) 



timalen Ergebnisse in der rekonstruktiven Chirurgie. Andererseits ist ein Chirurg, dem das Grundwissen in Anatomie, chirurgischen Techniken und der postoperativer Nachversorgung einschließlich der allfälligen Problembehandlung fehlt, kein sicherer Chirurg in der ästhetischen Chirurgie.

Glücklicherweise verbessern sich die Patientenergebnisse stetig, was zu einem gewissen Teil auf den internationalen Austausch von zeitgemäßen Konzepten zurückzuführen ist.

\title{
Korrespondenzadresse
}

B.F. Connell

2200 East Fruit Street Suite 101

CA 92701 Santa Ana

California

USA

bfclbca@aol.com

Abb. $2 \Delta$ a Fehlende definitive Endung des Tragus, b verdeckte intertragale Kerbe

Hautspannungen sind unnötig und verursachen Probleme bezüglich der Blutzufuhr zum Hautlappen sowie breite Narben. Exzisionen senkrecht zu einem ungeeigneten Vektor sowie exzessive Hautexzisionen heilen narbig ab. Ein unkorrekt lokalisierter Einschnitt resultiert in einer versetzten Haarlinie. Wie einem guten Chirurgen bewusst, beträgt der korrekte Winkel des Ohrläppchens über der Längsachse des Ohrs zwischen 11 und $15^{\circ}$. Jede Bewegung nach vorn mit dem Ohrläppchen anterior zur Längsachse lässt den $\mathrm{Pa}$ tienten aussehen, als habe er ein Facelifting vornehmen lassen (1960-1966). Ein weiterer Fehler bei mangelhaften anatomischen Kenntnissen sind eine fehlende definitive Endung des Tragus oder eine verdeckte intertragale Kerbe (• Abb. 2).

Durch den schnellen Wissensaustausch bei Konferenzen und Besuche bei herausragenden Chirurgen werden Kenntnisse vermittelt, die es erlauben, einen Patienten richtig zu bewerten und Techniken zur Vermeidung von Anzeichen eines chirurgischen Eingriffs zu erlernen. Somit kann das Ziel erreicht werden, den Patienten 18 oder 20 Jahre jünger aussehen zu lassen. Alle diese Faktoren, welche zu guten ästhetischen Ergebnissen führen, sind auch in der rekonstruktiven Chirurgie von wesentlicher Bedeutung. Ein Chirurg, der mit den Feinheiten der ästhetischen Chirurgie wenig vertraut ist, erzielt keine op-

\section{Hier steht eine Anzeige.}

\author{
炟 Springer
}

